\title{
The role of Lattice QCD in flavor physics
}

\author{
V. Lubicz ${ }^{\mathrm{a}}$ \\ a Dipartimento di Fisica, Università di Roma Tre and INFN, Sezione di Roma III \\ Via della Vasca Navale 84, I-00146 Rome, Italy \\ Understanding flavor physics is one of the most important tasks of particle physics today, which is motivating \\ an extraordinary experimental and theoretical investigational effort. Important progress in this field has already \\ been achieved in the last few years, with Lattice QCD calculations playing an essential role in this effort. I will \\ describe some lattice contributions to the studies of flavor physics by focusing particularly on the determination \\ of the CKM matrix and on the study of CP violation in the Standard Model.
}

\section{INTRODUCTION}

Flavor physics is the subject of intense experimental and theoretical research. There are well founded reasons for that, which can be summarized as follows:

- Flavor physics is (well) described but not explained in the Standard Model (SM). This is manifested by the large number of free parameters in the flavor sector of the SM (10 parameters in the quark sector only). In addition, many of these parameters, which in the SM Lagrangian enter as Yukawa couplings $y_{i j}$, assume unnaturally small values (at least in the mass diagonal basis). The fermion masses $m_{i} \sim y_{i i} v$, unlike the gauge boson masses $M_{W}$ and $M_{Z}$, are much smaller than the electroweak symmetry breaking scale $v$, with the notable exception of the top quark mass.

Besides providing us with an accurate parametric description, the SM does not explain flavor physics: we do not know why fermions come in families, nor what generates the hierarchy of masses, nor the quark mixing pattern encoded in the CKM matrix. In the leptonic sector, even the basic structure of the neutrino mass and mixing matrix is not established yet.

- In the absence of gauge symmetry breaking all particles in the SM remain massless and flavor symmetry is exact. The breaking of flavor symmetry (i.e. the generation of fermion masses) is originated by gauge symmetry breaking. The two mechanisms seem to be closely related and it may not be a coincidence that a definite explanation for both of them is lacking at present.

- Understanding the origin of $\mathrm{CP}$ violation is a fundamental issue in particle physics. This phenomenon has played a significant role in the primordial evolution of the Universe and it is expected to explain the origin of the observed asymmetry between matter and antimatter in the Universe. In the SM, the only source of $\mathrm{CP}$ violation is a single (physical) complex phase in the quark mass matrix. To verify the SM mechanism of CP violation is thus one of the most important tasks of current studies of flavor physics.

- It is beyond any reasonable doubt that the SM only represents the low energy limit of a more fundamental theory. Besides the well known conceptual problems of the SM, the most obvious of which is the non inclusion of a quantum description of gravity, there are important phenomenological indications of New Physics, i.e. physics beyond the SM, that have manifested in the last few years. The following is a (possibly incomplete) list of such indications. The existence of neutrino masses is experimentally well established, and the measured values of neutrino mass differences point to an energy scale which is close to the Grand Unification scale. Unification of couplings itself is indicated but not precisely achieved in the SM, thus suggesting the existence of New Physics at intermediate energy scales (below the GUT scale). At a cosmological level we find that vacuum energy represents a significant fraction of the total energy density of the Universe $\left(\Omega_{v a c} \sim 0.7\right)$ but this value is extraordinary small on a parti- 
cle physics energy scale, a phenomenon for which we have no convincing explanation. The rest of the energy density is mostly provided by matter $\left(\Omega_{\text {mat }} \sim 0.3\right)$, but the largest fraction of it (more than $80 \%$ ) is not of baryonic nature. Candidates for this dark matter are absent in the SM. Finally, the SM does not provide an acceptable explanation for baryogenesis (i.e. matter-antimatter asymmetry): the departure from thermal equilibrium is not sufficiently strong in the phase transition generated by electroweak symmetry breaking. Even most notably, the amount of $\mathrm{CP}$ violation originated by the CKM mechanism in the $\mathrm{SM}$ is not enough to explain baryogenesis.

It is likely that further insights in New Physics will eventually come from studying flavor physics. A simple naturalness argument in the SM, based on the requirement that radiative corrections to the Higgs boson mass should not exceed the upper bound on this mass deduced from electroweak precision tests, suggests that New Physics should appear at an energy scale of $\mathcal{O}(1 \mathrm{TeV})$. This scale is remarkably close to the region already explored by present experiments. On the other hand, upper bounds on New Physics coming from the flavor sector, e.g. from the value of the neutral kaon mass difference $\Delta M_{K}$, point to a much higher energy scale for New Physics, of $\mathcal{O}(100 \mathrm{TeV})$. The difficulty of explaining such a higher scale is sometimes called the flavor problem.

The above considerations illustrate the phenomenological and theoretical importance of the study of flavor physics. On the experimental side, both high-energy collision experiments and highstatistics dedicated flavor factories are providing us with results of increasing accuracy. In many relevant cases this accuracy has reached the level of $1 \%$ or better. On the theory side, one needs to match the precision achieved by experiments, which requires in particular an accurate determination of the theoretical input parameters. This is the present task of Lattice QCD calculations. In this talk I would like to show that we are already facing this task, and that Lattice QCD calculations play an essential role in present studies of flavor physics. I will focus the discussion on two main topics: i) the determination of the Cabibbo angle and ii) the analysis of the Unitarity Trian- gle and $\mathrm{CP}$ violation. An important contribution of Lattice QCD to the studies of flavor physics is represented also by the determination of quark masses, a topic which is reviewed by P. Rakow at this conference [1. Closely related to the subject of this discussion are the talk of M. Wingate [2] on the status of Lattice Flavor Physics and the stimulating "Experimenter's view of Lattice QCD" presented us by I. Shipsey [3].

\section{FIRST ROW UNITARITY}

The determination of the Cabibbo angle is of particular phenomenological and theoretical interest since it provides at present the most stringent unitarity test of the CKM matrix. This is expressed by the "first row" unitarity condition:

$\left|V_{u d}\right|^{2}+\left|V_{u s}\right|^{2}+\left|V_{u b}\right|^{2}=1$.

Since $\left|V_{u b}\right| \sim 10^{-3}$, its contribution to the Eq. (1) can be safely neglected.

The value of $\left|V_{u d}\right|$ is accurately determined from nuclear superallowed $0^{+} \rightarrow 0^{+}$and neutron beta decays. An updated analysis of these results leads to the very precise estimate [4]

$\left|V_{u d}\right|=0.9740 \pm 0.0005$,

where the average is dominated by superallowed transitions. In the following, I will therefore concentrate on the remaining entry $\left|V_{u s}\right|$.

\subsection{Present status of $\left|V_{u s}\right|$}

The most accurate determination of $\left|V_{u s}\right|$ is obtained from semileptonic kaon decays $\left(K_{\ell 3}\right)$. The analysis of the experimental data gives access to the quantity $\left|V_{u s}\right| \cdot f_{+}(0)$, where $f_{+}(0)$ is the vector form factor at zero four-momentum transfer square. In the $\mathrm{SU}(3)$ limit, vector current conservation implies $f_{+}(0)=1$. The deviation of $f_{+}(0)$ from unity represents the main source of theoretical uncertainty. This deviation has been estimated many years ago by Leutwyler and Roos (LR) [5], who combined a leading order analysis in chiral perturbation theory (ChPT) with a quark model calculation. They obtained $f_{+}^{K^{0} \pi^{-}}(0)=0.961 \pm 0.008$, and this value still represents the referential estimate [6].

By averaging old experimental results for $K_{\ell 3}$ decays with the recent measurement by E865 


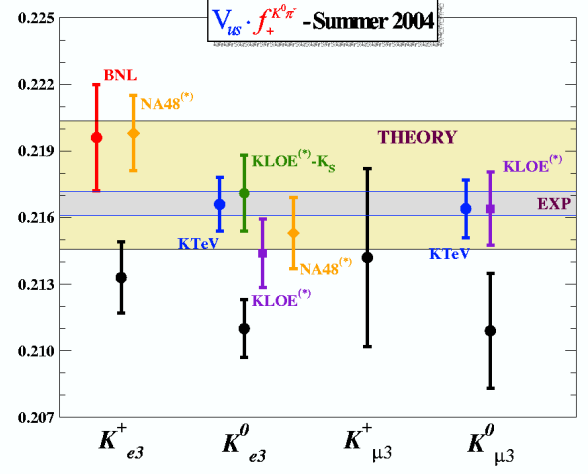

Figure 1. Experimental results for $\left|V_{u s}\right| \cdot f_{+}(0)$. The "EXP" and "THEORY" bands indicate respectively the average of the new experimental results and the unitarity prediction combined with the LR and lattice (see Sect[2.3) determination of the vector form factor.

at BNL [7, and using the LR determination of the vector form factor, the PDG quotes $\left|V_{u s}\right|=$ $0.2200 \pm 0.0026$ 6]. This value, once combined with the determination of $\left|V_{u d}\right|$ given in Eq. (2), implies about $2 \sigma$ deviation from the CKM unitarity condition, i.e. $\left|V_{u s}\right|^{\text {unit. }} \simeq \sqrt{1-\left|V_{u d}\right|^{2}}=$ $0.2265 \pm 0.0022$.

With respect to the PDG analysis, however, a significant novelty is represented by several new experimental results, for both charged and neutral $K_{\ell 3}$ decays, which have been recently presented by KTeV [8, NA48 9] and KLOE [10]. Expressed in terms of $\left|V_{u s}\right| \cdot f_{+}(0)$, these determinations are shown in Fig. 11 together with the BNL result and the averages of the old $K_{\ell 3}$ results quoted by the PDG. Remarkably, the average of the new results [11, represented by the darker band in the plot ("EXP"), is in very good agreement with the unitarity prediction, once the LR determination of the vector form factor is taken into account. The unitarity prediction is shown in Fig. 1 by the lighter band ("THEORY").

On the theoretical side, two important contributions to the determination of $\left|V_{u s}\right|$ come from lattice calculations and have been presented at this conference 1213. They concern leptonic and semileptonic kaon decays respectively. That already shows that Lattice QCD can contribute to precision calculations in flavor physics.

\section{2. $\left|\mathrm{V}_{\mathrm{us}}\right|$ from leptonic kaon decays}

As a part of their extensive study of partially quenched QCD with three dynamical flavors of improved staggered quarks, the MILC Collaboration has presented the final results for the light pseudoscalar decay constants [12 14,

$$
\begin{aligned}
f_{\pi} & =129.5 \pm 0.9 \pm 3.6 \mathrm{MeV}, \\
f_{K} & =156.6 \pm 1.0 \pm 3.8 \mathrm{MeV}, \\
f_{K} / f_{\pi} & =1.210 \pm 0.004 \pm 0.013,
\end{aligned}
$$

where the first error is statistical and the second systematic. The lattice data have been fit to staggered chiral perturbation theory expressions and extrapolated in quark masses, lattice spacing and lattice volume.

The uncertainties quoted in Eq. (3) represent an accuracy which has no precedent in lattice calculations of these quantities. It should be mentioned that there is a potential source of systematic error which is not quoted in Eq. (3) coming from the so called "fourth-root" trick applied to the staggered fermion determinant. A possibility exists that physical non-localities induced by this trick will remain in the continuum limit [14.

The precision achieved in Eq. (3) for $f_{K} / f_{\pi}$ allows for an accurate determination of $\left|V_{u s}\right| /\left|V_{u d}\right|$ from the ratio of leptonic kaon decay rates:

$\frac{\Gamma\left(K \rightarrow \mu \bar{\nu}_{\mu}(\gamma)\right)}{\Gamma\left(\pi \rightarrow \mu \bar{\nu}_{\mu}(\gamma)\right)}=K \frac{\left|V_{u s}\right|^{2} f_{K}^{2} m_{K}\left(1-\frac{m_{\mu}^{2}}{m_{K}^{2}}\right)^{2}}{\left|V_{u d}\right|^{2} f_{\pi}^{2} m_{\pi}\left(1-\frac{m_{\mu}^{2}}{m_{\pi}^{2}}\right)^{2}}$,

where $K=0.9930(35)$ takes into account radiative corrections [15]. By combining the experimental result $\Gamma\left(K \rightarrow \mu \bar{\nu}_{\mu}(\gamma)\right) / \Gamma\left(\pi \rightarrow \mu \bar{\nu}_{\mu}(\gamma)\right)=$ 1.334(4) with the Lattice QCD determination of $f_{K} / f_{\pi}$ in Eq. (3) and the value of $\left|V_{u d}\right|$ given in Eq. (2), one obtains

$\left|V_{u s}\right|=0.2219(1)(3)(4)(26)$.

The errors come respectively from the uncertainties on $\left|V_{u d}\right|$, the experimental measurement of leptonic decay rates, the radiative corrections in Eq. (4) and the lattice determination of $f_{K} / f_{\pi}$. The estimate of $\left|V_{u s}\right|$ in Eq. (5) is consistent with unitarity at the $1.4 \sigma$ level. Unfortunately, it will be difficult to further reduce the lattice uncertainty which clearly dominates the error. 


\section{3. $\left|\mathrm{V}_{\mathrm{us}}\right|$ from semileptonic kaon decays}

The other theoretical progress in the determination of $\left|V_{u s}\right|$ comes from the studies of semileptonic $K_{\ell 3}$ decays and it is represented by the first (quenched) lattice determination with significant accuracy of the vector form factor at zero momentum transfer square $f_{+}(0)$ [13 16]. The lattice result turns out to be in very good agreement with the quark model estimate obtained by LR, thus putting the evaluation of this form factor on a firmer theoretical basis. Before outlining the strategy of the lattice calculation, whose details have been presented by F. Mescia at this conference [13, I would like to summarize the theoretical status of the $f_{+}(0)$ evaluations.

A good theoretical control on $K_{\ell 3}$ transitions is assured by the Ademollo-Gatto (AG) theorem [17, which states that $f_{+}(0)$ is renormalized only by terms of at least second order in the breaking of SU(3)-flavor symmetry. Nevertheless, the error on the shift of $f_{+}(0)$ from unity represents not only the main source of theoretical uncertainty but it also dominates the overall error in the determination of $\left|V_{u s}\right|$.

The amount of $\mathrm{SU}(3)$ breaking due to light quark masses can be investigated within ChPT, by performing an expansion of the form $f_{+}(0)=$ $1+f_{2}+f_{4}+\ldots$, where $f_{n}=\mathcal{O}\left(p^{n}\right)=$ $\mathcal{O}\left[M_{K, \pi}^{n} /\left(4 \pi f_{\pi}\right)^{n}\right]$. Thanks to the AG theorem, the first non-trivial term in the chiral expansion, $f_{2}$, does not receive contributions of local operators appearing in the effective theory and can be computed unambiguously in terms of $M_{K}, M_{\pi}$ and $f_{\pi}\left(f_{2}=-0.023\right.$, in the $K^{0} \rightarrow \pi^{-}$case 5] ). The higher-order terms of the chiral expansion involve instead the coefficients of local chiral operators, that are difficult to estimate. The quark model calculation by LR provides an estimate of the next-to-leading correction $f_{4}$, and it is based on a general parameterization of the $\mathrm{SU}(3)$ breaking structure of the pseudoscalar meson wave functions.

An important progress in this study is represented by the complete two-loop ChPT calculation of $f_{4}$, performed in Refs. 1819. The result can be written in the form

$f_{4}=\Delta(\mu)-\frac{8}{F_{\pi}^{4}}\left[C_{12}(\mu)+C_{34}(\mu)\right]\left(M_{K}^{2}-M_{\pi}^{2}\right)^{2},(6)$ where $\Delta(\mu)$ represents the loop contribution, expressed in terms of chiral logs and the $\mathcal{O}\left(p^{4}\right)$ lowenergy constants, while the second term is the analytic one. As can be seen from Eq. (6), the local contribution involves a single combination of two (unknown) chiral coefficients entering the effective Lagrangian at $\mathcal{O}\left(p^{6}\right)$. In addition, the separation between non-local and local contribution quantitatively depends on the choice of the renormalization scale $\mu$, only the whole result for $f_{4}$ being scale independent. This dependence is found to be large 20]; for instance, at three typical values of the scale one finds

$\Delta(\mu)=\left\{\begin{array}{ccc}0.031, & 0.015, & 0.004 \\ \mu=M_{\eta} & \mu=M_{\rho}\end{array} \quad \mu=1 \mathrm{GeV}\right.$.

An important observation by Bijnens and Talavera [19] is that the combination of low-energy constants entering $f_{4}$ could be in principle constrained by experimental data on the slope and curvature of the scalar form factor. The required level of experimental precision, however, is far from what is currently achieved. Thus, one is left with either the LR result or other model dependent estimates of the local term in Eq. (6). Recent attempts in this direction include the estimate by resonance saturation obtained in Ref. 20 and the dispersive analysis of Ref. 21. On the other hand, the large scale dependence of the $\mathcal{O}\left(p^{6}\right)$ loop calculation shown in Eq. (7) seems to indicate that the error \pm 0.010 quoted in Refs. [19]21 might be underestimated. A first principle lattice determination of the vector form factor is thus of great phenomenological relevance.

In order to reach the challenging goal of about $1 \%$ error on the lattice determination of $f_{+}(0)$, a new strategy has been proposed and applied in the quenched approximation 16. This strategy is based on three steps.

1) Precise evaluation of $f_{0}\left(q^{2}\right)$ at $q^{2}=q_{\max }^{2}$ This evaluation follows a procedure originally proposed by the FNAL group to study heavylight form factors 22 . For $K_{\ell 3}$ decays, the scalar form factor $f_{0}\left(q^{2}\right)$ can be calculated very efficiently at $q^{2}=q_{\max }^{2}=\left(M_{K}-M_{\pi}\right)^{2}$ by studying the following double ratio of matrix elements,

$\frac{\left\langle\pi\left|\bar{s} \gamma_{0} u\right| K\right\rangle\left\langle K\left|\bar{u} \gamma_{0} s\right| \pi\right\rangle}{\left\langle K\left|\bar{s} \gamma_{0} s\right| K\right\rangle\left\langle\pi\left|\bar{u} \gamma_{0} u\right| \pi\right\rangle}=C_{K, \pi}\left[f_{0}\left(q_{\max }^{2}\right)\right]^{2}$, 

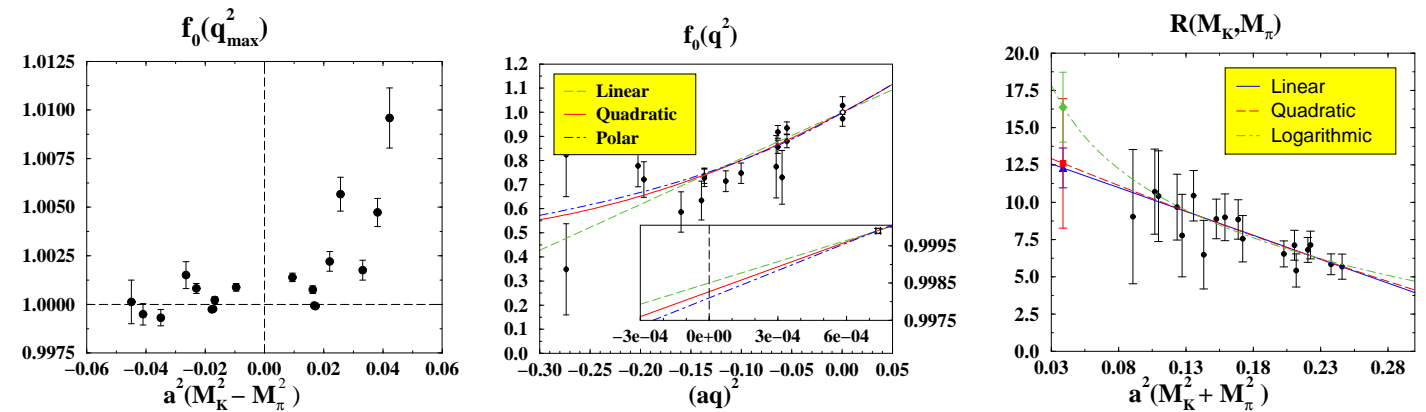

Figure 2. Left: Values of $f_{0}\left(q_{\max }^{2}\right)$ versus the $S U(3)$-breaking parameter $a^{2}\left(M_{K}^{2}-M_{\pi}^{2}\right)$. Center: The form factor $f_{0}\left(q^{2}\right)$ as a function of $q^{2}$ for one of the quark mass combinations used in the simulation. The inset is an enlargement of the region around $q^{2}=0$. Right: Comparison among linear, quadratic and logarithmic chiral extrapolations of the ratio $R$ of Eq. (9) as a function of $a^{2}\left(M_{K}^{2}+M_{\pi}^{2}\right)$.

where all the external particles are taken at rest and $C_{K, \pi}=\left(M_{K}+M_{\pi}\right)^{2} /\left(4 M_{K} M_{\pi}\right)$. There are several crucial advantages in using the double ratio (8) which are described in details in Ref. [16. The most important point is that this ratio gives values of $f_{0}\left(q_{\max }^{2}\right)$ with a statistical uncertainty smaller than 0.1\%, as shown in Fig. 2 (left).

2) Extrapolation of $\mathbf{f}_{\mathbf{0}}\left(\mathbf{q}_{\max }^{2}\right)$ to $\mathbf{f}_{\mathbf{0}}(\mathbf{0})=\mathbf{f}_{+}(\mathbf{0})$ This extrapolation is performed by studying the $q^{2}$-dependence of $f_{0}\left(q^{2}\right)$. New suitable double ratios are introduced also in this step, that improve the statistical accuracy of $f_{0}\left(q^{2}\right)$. The quality of the extrapolation is shown in Fig. 2 (center). Three different functional forms in $q^{2}$ have been considered, namely a polar, a linear and a quadratic one. The differences among the results are considered in the evaluation of the systematic error.

\section{3) Extrapolation to the physical masses}

The physical value of $f_{+}(0)$ is finally reached after extrapolating the lattice results to the physical kaon and pion masses. The problem of the chiral extrapolation is substantially simplified if the AG theorem is taken into account and if the leading (quenched) chiral logs are subtracted. This is achieved by introducing the following ratio

$R=\frac{\Delta f}{\left(M_{K}^{2}-M_{\pi}^{2}\right)^{2}}=\frac{1+f_{2}^{q}-f_{+}(0)}{\left(M_{K}^{2}-M_{\pi}^{2}\right)^{2}}$,

where $f_{2}^{q}$ represents the leading chiral contribution calculated in quenched ChPT [16] and the quadratic dependence on $\left(M_{K}^{2}-M_{\pi}^{2}\right)$, driven by the AG theorem, is factorized out. It should be emphasized that the subtraction of $f_{2}^{q}$ in Eq. (9) does not imply necessarily a good convergence of (quenched) $\mathrm{ChPT}$ at $\mathcal{O}\left(p^{4}\right)$ for the meson masses used in the lattice simulation. The aim of the subtraction is to access directly on the lattice the quantity $\Delta f$, defined in such a way that its chiral expansion starts at $\mathcal{O}\left(p^{6}\right)$ independently of the values of the meson masses. After the subtraction of $f_{2}^{q}$, the ratio $R$ of Eq. (9) is smoothly extrapolated in the meson masses as illustrated in Fig. 2 (right). In order to check the stability of the extrapolation, linear, quadratic and logarithmic fits have been considered. The leads to the final result

$f_{+}^{K^{0} \pi^{-}}(0)=0.960 \pm 0.005_{\text {stat }} \pm 0.007_{\text {syst }}$,

where the systematic error does not include quenching effects beyond $\mathcal{O}\left(p^{4}\right)$. Removing this error represents one of the major goal of future lattice studies of $K_{\ell 3}$ decays.

The value (10) compares well with the LR result $f_{+}^{K^{0} \pi^{-}}(0)=0.961 \pm 0.008$ quoted by the PDG 6] and, once combined with the average of the more recent experimental results, implies

$\left|V_{u s}\right|=0.2256 \pm 0.0022$,

in good agreement with unitarity.

A strategy similar to the one discussed above has been also applied to study hyperon semileptonic decays on the lattice, and preliminary results have been presented at this conference 23 . 
Table 1

Central values and errors of some of the most relevant input quantities used in the UT analysis.

\begin{tabular}{ll}
\hline \hline Parameter & Value \\
\hline \hline$\lambda$ & $0.2265 \pm 0.0020$ \\
\hline$\left|V_{c b}\right|($ excl. $)$ & $(42.1 \pm 2.1) \times 10^{-3}$ \\
$\left|V_{c b}\right|($ incl. $)$ & $(41.4 \pm 0.7 \pm 0.6) \times 10^{-3}$ \\
$\left|V_{u b}\right|($ excl. $)$ & $(33.0 \pm 2.4 \pm 4.6) \times 10^{-4}$ \\
$\left|V_{u b}\right|$ (incl.-LEP) & $(40.9 \pm 6.2 \pm 4.7) \times 10^{-4}$ \\
$\left|V_{u b}\right|($ incl.-HFAG $)$ & $(45.7 \pm 6.1) \times 10^{-4}$ \\
\hline$\Delta m_{d}$ & $(0.503 \pm 0.006) \mathrm{ps}^{-1}$ \\
$\Delta m_{s}$ & $>14.5 \mathrm{ps}^{-1}$ at $95 \%$ C.L. \\
& sensitivity $18.3 \mathrm{ps}^{-1}$ \\
$\sin 2 \beta$ & $0.739 \pm 0.048$ \\
\hline$f_{B_{s}} \hat{B}_{B_{s}}^{1 / 2}$ & $276 \pm 38 \mathrm{MeV}$ \\
$\xi=f_{B_{s}} \hat{B}_{B_{s}}^{1 / 2} / f_{B_{d}} \hat{B}_{B_{d}}^{1 / 2}$ & $1.24 \pm 0.04 \pm 0.06$ \\
$\hat{B}_{K}$ & $0.86 \pm 0.06 \pm 0.14$ \\
\hline \hline
\end{tabular}

\section{LATTICE QCD AND THE UNITARI- TY TRIANGLE ANALYSIS}

Now, I would like to illustrate the role of Lattice QCD calculations in providing the input parameters to the analysis of the Unitarity Triangle (UT) and of CP violation. This study provides one of the most significant tests of flavor physics in the SM and a unique opportunity for searching New Physics.

\subsection{Lattice input parameters}

The most precise determination of the UT parameters is obtained by using semileptonic Bdecays, $\mathrm{B}^{0}-\overline{\mathrm{B}^{0}}$ oscillations and $\mathrm{CP}$ asymmetries in the kaon and in the B sectors. This will be referred to as the "standard analysis" 24- 28, and relies on the following five measurements: the ratio $\left|V_{u b}\right| /\left|V_{c b}\right|$, the mass differences in the neutral B-meson systems, $\Delta m_{d}$ and the limit on $\Delta m_{s}$, and the CP-violating quantities in the kaon $\left(\varepsilon_{K}\right)$ and in the $\mathrm{B}(\sin 2 \beta)$ sectors. Lattice QCD calculations play a central role by determining many of the theoretical inputs. A list of the most relevant input quantities, with corresponding central values and errors, is given in Table 11. The main input which come from lattice calculations

${ }^{1}$ See http://www.utfit.org for the complete collection of input quantities and results. are the form factors controlling semileptonic Bdecays, the hadronic parameters $f_{B_{s}} \hat{B}_{B_{s}}^{1 / 2}$ and $\xi$ entering the amplitudes of $\mathrm{B}^{0}-\overline{\mathrm{B}^{0}}$ oscillations, and the bag parameter $\hat{B}_{K}$ which parameterizes $\mathrm{K}^{0}-\overline{\mathrm{K}^{0}}$ mixing. The central values and errors quoted in Table 1 are those adopted at the end of the "CKM Unitarity Triangle" workshops 2930 and by the HFAG 31. With respect to previous analyses [24 25], the novelties in Table 1 are the final LEP/SLD likelihood for $\mathrm{B}_{s}$ oscillations, the value of $\left|V_{u b}\right|$ from inclusive semileptonic decays 31, the new value of $\sin 2 \beta$ and a new treatment of the non-perturbative QCD parameters controlling $\mathrm{B}^{0}-\overline{\mathrm{B}^{0}}$ mixing, as explained below.

In previous analyses, the constraints coming from the experimental informations on $\Delta m_{d}$ and $\Delta m_{s}$ were implemented as

$\Delta m_{d} \propto\left|V_{t d}\right|^{2} f_{B_{d}}^{2} \hat{B}_{B_{d}} \propto\left[(1-\bar{\rho})^{2}+\bar{\eta}^{2}\right] f_{B_{d}}^{2} \hat{B}_{B_{d}}$

$\Delta m_{s} \propto\left|V_{t s}\right|^{2} f_{B_{s}}^{2} \hat{B}_{B_{s}} \propto f_{B_{d}}^{2} \hat{B}_{B_{d}} \times \xi^{2}$

In this case, the input quantities are $f_{B_{d}} \hat{B}_{B_{d}}^{1 / 2}$ and $\xi$. The hadronic parameter that is better determined from lattice calculations, however, is $f_{B_{s}} \hat{B}_{B_{s}}^{1 / 2}$, whereas $\xi$ and $f_{B_{d}} \hat{B}_{B_{d}}^{1 / 2}$ are affected by larger uncertainties coming from the chiral extrapolations. These uncertainties are strongly correlated. For this reason, a better approach consists in writing the constraints as follows:

$\begin{aligned} \Delta m_{d} & \propto\left[(1-\bar{\rho})^{2}+\bar{\eta}^{2}\right] f_{B_{s}}^{2} \hat{B}_{B_{s}} / \xi^{2} \\ \Delta m_{s} & \propto f_{B_{s}}^{2} \hat{B}_{B_{s}}\end{aligned}$

At present, this new parameterization does not have a large effect on final results. It allows, however, to take better into account the uncertainty from the chiral extrapolation in lattice calculations of $f_{B_{d}}$.

\subsection{Statistical methods}

The two statistical methods most often used in current UT analyses are the Bayesian [24]- 26] and the frequentistic [27]-28] approach.

The Bayesian approach is based on the Bayes' theorem, which relates the probability of an event $A$, given the event $B$, to the probability of $B$ given $A$ :

$P(A \mid B)=P(B \mid A) \cdot P(A) / P(B)$. 
In the UT analysis, the event $A$ is represented by given values of the UT parameters, $\bar{\rho}$ and $\bar{\eta}$, and of all the other parameters, denoted by $\mathbf{x}$, entering the analysis (the Cabibbo angle, $\left|V_{c b}\right|$, the top quark mass, $\hat{B}_{K}$, etc.). The event $B$ represents instead the whole set of experimental constraints (the measurements of $\varepsilon_{K}, \Delta m_{d}, \sin 2 \beta$, etc.), denoted collectively by c. The Bayes' theorem (14) then allows one to compute the probability of given values of $\bar{\rho}, \bar{\eta}$ and $\mathbf{x}$ given the experimental constraints:

$f(\bar{\rho}, \bar{\eta}, \mathbf{x} \mid \mathbf{c}) \propto f(\mathbf{c} \mid \bar{\rho}, \bar{\eta}, \mathbf{x}) \cdot f_{0}(\mathbf{x}) f_{0}(\bar{\rho}, \bar{\eta})$.

The function $f_{0}(\mathbf{x})$ is the so called a priori probability of $\mathbf{x}$. It expresses our knowledge on these parameters, coming for instance from Lattice QCD calculations. As for the a priori probability $f_{0}(\bar{\rho}, \bar{\eta})$, this is usually represented by a large flat distribution, since nothing is known about $\bar{\rho}$ and $\bar{\eta}$ before the UT analysis is performed. Integrating both sides of Eq. (15) over all possible values of $\mathbf{x}$, finally leads to

$f(\bar{\rho}, \bar{\eta} \mid \mathbf{c}) \propto \mathcal{L}(\mathbf{c} \mid \bar{\rho}, \bar{\eta}) \cdot f_{0}(\bar{\rho}, \bar{\eta})$.

The function $\mathcal{L}(\mathbf{c} \mid \bar{\rho}, \bar{\eta})$ is the total likelihood.

There are several differences between the Bayesian and the frequentistic approach, the most significant of which is the assumption within the latter that an a priori probability distribution function (p.d.f.) cannot be defined for theoretical parameters. As a consequence, in the frequentistic approach the likelihood is defined in such a way that its "theoretical" part does not contribute to the fit while the corresponding parameters take values within "allowed" ranges. In more recent versions of the frequentistic approach the above statement is applied in practice only to the systematic part of the uncertainties quoted for the theoretical parameters. Figure 3 shows, as an example, the $\Delta$-likelihood for the parameter $\hat{B}_{K}$ as obtained in the Bayesian and in the frequentistic approach using $\hat{B}_{K}=0.86 \pm 0.06 \pm 0.14$.

As a member of a Bayesian collaboration, I do not need to specify which of the two approaches I do prefer. What I like about the Bayesian approach is that, being based on clear assumptions, it allows one to reach conclusions with a well defined statistical meaning. This is not the

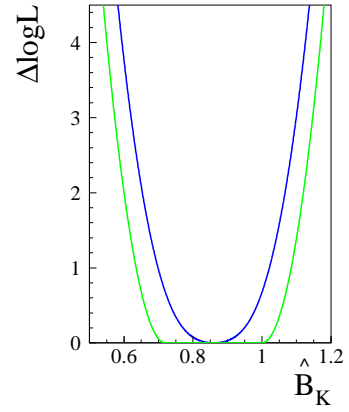

Figure 3. The $\Delta$-likelihood for $\hat{B}_{K}$ as obtained in the Bayesian (dark curve) and frequentistic (light curve) approach using $\hat{B}_{K}=0.86 \pm 0.06 \pm 0.14$.

case for the frequentistic approach, in which final results are usually quoted as having at least $95 \%$ of probability. The point I would most like to emphasize however is: irrespective of the preferred statistical method, the contribution to the likelihood coming from the knowledge of a given theoretical parameter, like $\hat{B}_{K}$ in Fig. 3 should be specified by the people who calculated it, rather than by those who perform the UT analysis. In the specific example, it is our task in the lattice community to specify what we mean by " $\hat{B}_{K}=0.86 \pm 0.06 \pm 0.14$ ". In this way, the difference expressed by the two curves in Fig. 3 would be resolved.

\subsection{Results for the UT parameters}

The most significant test of the CKM mechanism of $\mathrm{CP}$ violation is the comparison between the $(\bar{\rho}, \bar{\eta})$ region selected by the measurements of $\mathrm{CP}$ conserving quantities (semileptonic B decays and $\mathrm{B}^{0}-\overline{\mathrm{B}^{0}}$ oscillations) and the regions selected by the measurements of $\mathrm{CP}$ violation in the kaon $\left(\varepsilon_{K}\right)$ or in the $\mathrm{B}(\sin 2 \beta)$ sectors.

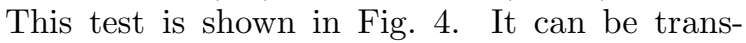
lated quantitatively by the comparison between the value of $\sin 2 \beta$ determined from the UT analysis when only $\mathrm{CP}$ conserving quantities are included ("sides" measurements) and the one obtained from the CP asymmetry measurement in $B \rightarrow J / \psi K_{s}$ decays:

$$
\begin{array}{ll}
\sin 2 \beta=0.724 \pm 0.049 & \text { Sides only } \\
\sin 2 \beta=0.739 \pm 0.048 & B \rightarrow J / \psi K_{s} .
\end{array}
$$

The excellent agreement between these values illustrates the consistency of the SM in describing 


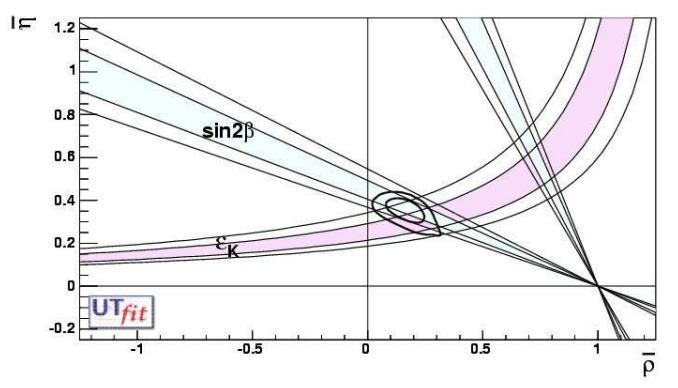

Figure 4. Comparison between the allowed regions for $\bar{\rho}$ and $\bar{\eta}$ (at $68 \%$, 95\% probability ranges) selected by measurements of the CP conserving quantities and the bands derived from the measurements of $C P$ violating quantities in the kaon $\left(\varepsilon_{K}\right)$ and in the $B(\sin 2 \beta)$ sectors.

Table 2

Values of the main UT parameters obtained from the standard analysis.

\begin{tabular}{cc}
\hline \hline Parameter & \\
\hline $\bar{\eta}$ & $0.348 \pm 0.028$ \\
$\bar{\rho}$ & $0.172 \pm 0.047$ \\
$\sin 2 \beta$ & $0.725 \pm 0.033$ \\
$\sin 2 \alpha$ & $-0.16 \pm 0.26$ \\
$\gamma\left[^{\circ}\right]$ & $61.5 \pm 7.0$ \\
\hline \hline
\end{tabular}

$\mathrm{CP}$ violation phenomena in terms of one single parameter $\bar{\eta}$.

The results for the main UT parameters obtained from the standard analysis are presented in Table 2] Figures 5 and 6 show respectively some of the corresponding p.d.f.'s and the selected region in the $(\bar{\rho}, \bar{\eta})$ plane.

Another interesting prediction of the UT analysis concerns the mass difference $\Delta m_{s}$. This prediction is obtained by removing from the fit the experimental information coming from $\mathrm{B}_{s}^{0}-\overline{\mathrm{B}_{\mathrm{s}}^{0}}$ oscillations (present analyses at LEP/SLD have established a sensitivity of $18.3 \mathrm{ps}^{-1}$ and they show a higher probability region for a positive signal around $17.5 \mathrm{ps}^{-1}$ ). The prediction is

$\Delta m_{s}=(21.1 \pm 3.1) \mathrm{ps}^{-1}$.

It is also interesting to quote the value obtained when the experimental information on $\mathrm{B}_{s}^{0}-\overline{\mathrm{B}_{\mathrm{s}}^{0}}$ oscillations is included in the analysis,

$\Delta m_{s}=(18.3 \pm 1.6) \mathrm{ps}^{-1} \quad$ (including $\left.\Delta m_{s}\right) .(19)$
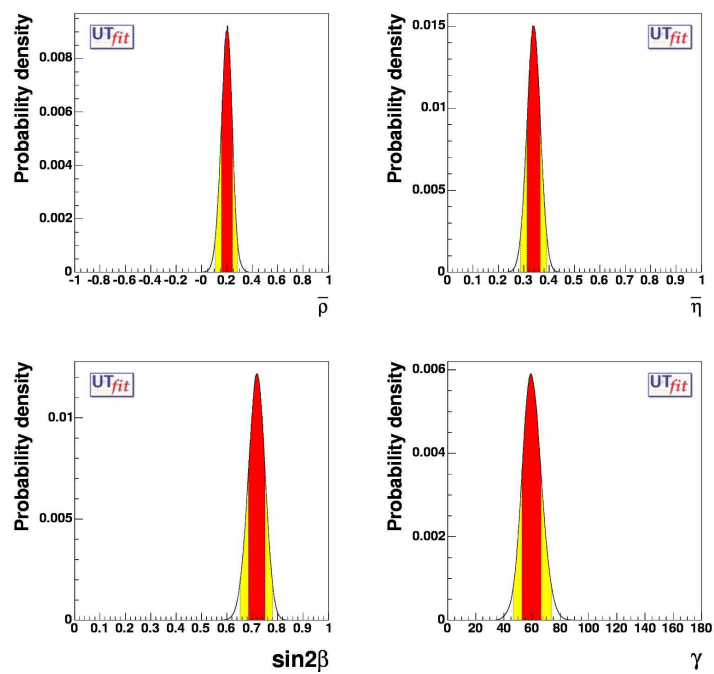

Figure 5. P.d.f.'s for $\bar{\rho}, \bar{\eta}, \sin 2 \beta$ and $\gamma$. The red (dark) and the yellow (light) zones correspond respectively to $68 \%$ and $95 \%$ of probability.

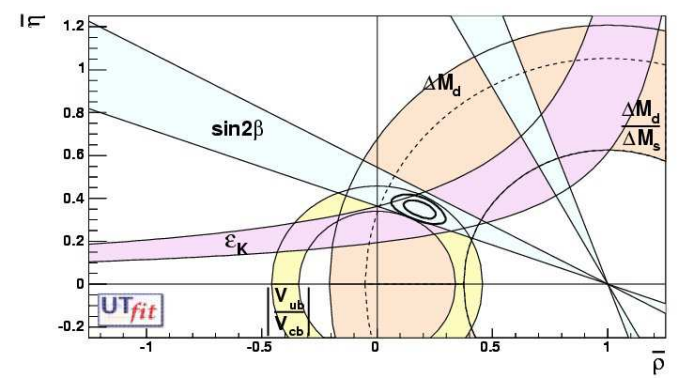

Figure 6. Allowed regions (contours at 68\% and $95 \%$ probability) for $\bar{\rho}$ and $\bar{\eta}$. The bands show the 95\% probability regions selected from the various constraints.

Once an accurate measurement of $\Delta m_{s}$, expected from the TeVatron in the near future, will become available, the prediction of Eq. (18) will provide a crucial test of the SM.

\subsection{Hadronic parameters}

A study of particular interest from the Lattice QCD point of view is the determination of the hadronic parameters from the UT analysis. This study consists of removing from the analysis, input information coming from Lattice QCD, namely the estimates of $f_{B_{s}} \hat{B}_{B_{s}}^{1 / 2}, \xi$ and $\hat{B}_{K}$, by treating these quantities as free parameters in the fit. In this way, the result obtained from the UT 
Table 3

Values of the non-perturbative QCD parameters as obtained from the UT analysis. The current Lattice QCD determinations are also shown for comparison.

\begin{tabular}{c|c|c}
\hline \hline Parameter & From UT fit & From Lattice QCD \\
\hline$\hat{B}_{K}$ & $0.65 \pm 0.10$ & $0.86 \pm 0.06 \pm 0.14$ \\
\hline$f_{B_{s}} \hat{B}_{B_{s}}^{1 / 2}$ & $263 \pm 14 \mathrm{MeV}$ & $276 \pm 38 \mathrm{MeV}$ \\
\hline$\xi$ & $1.13_{-0.09}^{+0.12}$ & $1.24 \pm 0.04 \pm 0.06$ \\
\hline \hline
\end{tabular}

fit for a given hadronic parameter can be compared to the corresponding lattice determination. Table 3 shows the results of the fit when one hadronic parameter at a time is treated as a free parameter. The current Lattice QCD determinations are shown for comparison.

Some conclusions can be drawn. The precision on $f_{B_{s}} \hat{B}_{B_{s}}^{1 / 2}$ obtained from the UT fit has an accuracy which is better than the current Lattice QCD estimate. This proves that the standard CKM fit is, in practice, weakly dependent on the theoretical uncertainty on this parameter. The UT fit result for $\hat{B}_{K}$ indicates that values of $\hat{B}_{K}$ smaller than 0.45 are excluded at $99 \%$ probability. The accuracy achieved in the estimate of $\hat{B}_{K}$ from the fit is at the level of $15 \%$, comparable to the one obtained from Lattice QCD. On the other hand, the best determination of the parameter $\xi$ comes at present from the lattice.

\subsection{Impact of improved determinations}

Before concluding this section I would like to illustrate to what extent the UT analysis could benefit from more accurate lattice calculations in the next few years. I assume that the uncertainties in the values of the lattice input parameters will be reduced as indicated in Table 4 to be compared with current uncertainties presented in Table1 A discussion on the extent to which such a projection is realistic is beyond the scope of this paper. Besides reducing the uncertainties in the hadronic parameters, I take into account the impact of a more accurate experimental determination of $\sin 2 \beta$ from $B \rightarrow J / \psi K_{s}$ decays.

The region in the $(\bar{\rho}, \bar{\eta})$ plane selected by the analysis with the projection for the near future is shown in Fig. [7 which is to be compared
Table 4

Values of the input parameters used in the UT analysis in the projection for the next years.

\begin{tabular}{c|c}
\hline \hline Parameter & Next years \\
\hline$\hat{B}_{K}$ & $0.86 \pm 0.06$ \\
$f_{B_{s}} \hat{B}_{B_{s}}^{1 / 2}$ & $276 \pm 14 \mathrm{MeV}$ \\
$\xi$ & $1.24 \pm 0.04$ \\
$\left|V_{u b}\right|($ excl. $)$ & $(33.0 \pm 2.4) \times 10^{-4}$ \\
$\sin 2 \beta$ & $0.739 \pm 0.021$ \\
\hline \hline
\end{tabular}

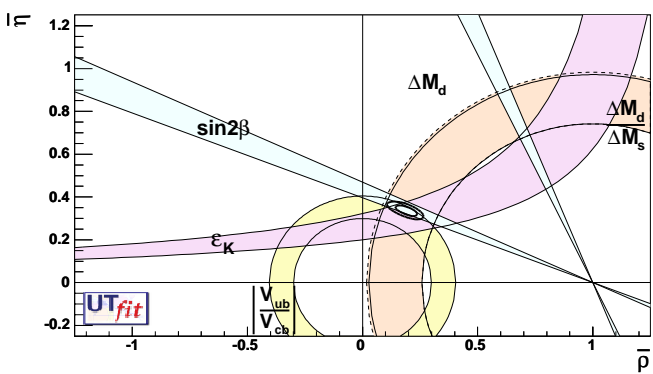

Figure 7. The contours at $68 \%$ and $95 \%$ probability in the $(\bar{\rho}, \bar{\eta})$ plane as obtained in the projection for the next years.

with the present determination shown in Fig. [6] Quantitatively, the effect is a reduction of the uncertainties on both $\bar{\rho}$ and $\bar{\eta}$ by approximately $40 \%$ (from $27 \%$ to $17 \%$ for $\bar{\rho}$ and from $8 \%$ to $5 \%$ for $\bar{\eta})$. In achieving this result, the assumption of a $5 \%$ accuracy reached in the determination of $f_{B_{s}} \hat{B}_{B_{s}}^{1 / 2}$ plays a minor role since, as discussed before, the value of this parameter is already quite constrained by the UT fit. On the other hand, the precision in the determination of $f_{B_{s}} \hat{B}_{B_{s}}^{1 / 2}$ has a crucial impact on the prediction of the mass difference $\Delta m_{s}$. The value obtained from the projection for the future is

$\Delta m_{s}=(19.7 \pm 1.8) \mathrm{ps}^{-1}$,

to be compared with the present determination quoted in Eq. (18).

\section{CONCLUSIONS}

Flavor physics in the quark sector has entered its mature age. The precision reached in the determination of the fundamental parameters, namely quark masses and CKM matrix elements, has significantly improved. The mixing between 
the first two generations of quarks is accurately determined, and recent experimental results on $K_{\ell 3}$ decays, combined with the precise theoretical determination of the relevant form factor, provide a value of $\left|V_{u s}\right|$ in excellent agreement with the unitarity prediction. The UT parameters, which define the mixing between the first and the third generations, are determined with good precision. A crucial test of the CKM mechanism of CP violation has already been performed, namely the comparison between the UT parameters as determined from $\mathrm{CP}$ conserving quantities (semileptonic $\mathrm{B}$ decays and $\mathrm{B}^{0}-\overline{\mathrm{B}^{0}}$ oscillations), and the measurements of $\mathrm{CP}$ violation in the kaon $\left(\epsilon_{K}\right)$ and in the $\mathrm{B}(\sin 2 \beta)$ sectors. The agreement is excellent. Such a test could not have been performed without the essential contribution of Lattice QCD calculations.

Though I did not have time to discuss them in this talk, it should be mentioned that measurements of non-leptonic B meson decays at the B-Factories have already started to have an impact on the UT analysis 26]. In the next years, these measurements will allow tests of the SM in the flavor sector to an accuracy up to the per cent level. A crucial role will be also played by precision charm physics. Accurate measurements of leptonic and semileptonic D-meson decay rates will be available soon from CLEO-c [3], allowing for precise determinations of the CKM parameters $V_{c s}$ and $V_{c d}$. In this important research, Lattice QCD calculations are expected to provide the necessary theoretical support.

\section{ACKNOWLEDGMENTS}

I wish to thank C. Bernard, P. Gambino, L. Giusti, G. Isidori, M. Wingate, S. Sharpe and I. Shipsey for useful discussions. I am grateful to A. Kronfeld and E. Freeland for a careful reading and for valuable suggestions on the manuscript. I am also grateful to all my friends and colleagues of the SPQ ${ }_{C D} R$ and UTFit Collaborations for an enjoyable, fruitful and longstanding collaboration.

\section{REFERENCES}

1. P. Rakow, these Proceedings.
2. M. Wingate, these Proceedings.

3. I. Shipsey, these Proceedings.

4. A. Czarnecki et al., hep-ph/0406324

5. H. Leutwyler and M. Roos, Z. Phys. C 25 (1984) 91.

6. S. Eidelman et al. [Particle Data Group Collaboration], Phys.Lett.B 592 (2004) 1.

7. A. Sher et al., Phys. Rev. Lett. 91, 261802 (2003) hep-ex/0305042.

8. T. Alexopoulos et al., hep-ex/0406001.

9. L. Litov, presented at ICHEP'04.

10. P. Franzini, hep-ex/0408150 M. Antonelli, presented at ICHEP'04.

11. F. Mescia, presented at ICHEP'04.

12. C. Aubin et al., these Proceedings

13. D. Becirevic et al., these Proceedings.

14. C. Aubin et al., hep-lat/0407028

15. W. J. Marciano, hep-ph/0402299

16. D. Becirevic et al., hep-ph/0403217

17. M. Ademollo and R. Gatto, Phys. Rev. Lett. 13, 264 (1964).

18. P. Post and K. Schilcher, Eur. Phys. J. C 25, 427 (2002) hep-ph/0112352.

19. J. Bijnens and P. Talavera, Nucl. Phys. B 669, 341 (2003) hep-ph/0303103.

20. V. Cirigliano, H. Neufeld and H. Pichl, Eur. Phys. J. C 35 (2004) 53 hep-ph/0401173.

21. M. Jamin, J. A. Oller and A. Pich, JHEP 0402 (2004) 047 hep-ph/0401080.

22. S. Hashimoto et al., Phys. Rev. D 61 (2000) 014502 hep-ph/9906376.

23. D. Guadagnoli et al., these Proceedings.

24. M. Ciuchini et al., JHEP 0107 (2001) 013 hep-ph/0012308.

25. M. Ciuchini et al., eConf C0304052, WG306 (2003) hep-ph/0307195.

26. M. Bona et al., hep-ph/0408079

27. A. Hocker et al., Eur. Phys. J. C 21 (2001) 225 hep-ph/0104062.

28. J. Charles et al., hep-ph/0406184

29. Proceedings of the First Workshop on the CKM Unitarity Triangle, CERN 2002, edited by M. Battaglia et al., hep-ph/0304132.

30. Proceedings of the Second Workshop on the CKM Unitarity Triangle, Durham, 2003, ed. by P. Ball et al., eConf C0304052 (2003).

31. The Heavy Flavor Averaging Group (HFAG), http://www.slac.stanford.edu/xorg/hfag/ 